\title{
An approach to Fuzzy clustering of the iris petals by using $\mathcal{A c}$-means
}

\author{
Nicolás Enrique Salgado Guitiérrez ${ }^{1}$, Sergio Andrés Valencia Ramírez ${ }^{2}$ \\ and José Soriano Méndez ${ }^{3}$ \\ ${ }^{1}$ Engineering department, District University FJDC, Bogota D.C., 110231, Bogota, Colombia \\ nesalgadog@correo.udistrital.edu.co \\ ${ }^{2}$ Engineering department, District University FJDC, Bogota D.C., 110231, Bogota, Colombia \\ savalenciar@correo.udistrital.edu.co \\ ${ }^{3}$ Engineering department, District University FJDC, Bogota D.C., 110231, Bogota, Colombia, Advisor
}

\begin{abstract}
This paper proposes a definition of a fuzzy partition element based on the homomorphism between type-1 fuzzy sets and the three-valued Kleene algebra. A new clustering method based on the C-means algorithm, using the defined partition, is presented in this paper, which will be validated with the traditional iris clustering problem by measuring its petals.
\end{abstract}

Keywords Fuzzy, Partition, $\mathcal{K}_{3}$ algebra, homomorphism, cluster, C-means.

\section{Introduction}

The iris clustering problem has been approached from different perspectives, which perform such clustering under certain common characteristics represented by metrics.

These perspectives are described with many types of algorithms. One of the most commonly used is C-means, which consists of creating fuzzy partitions according to the formal definition of Ruspini[1]. This algorithm is an extension of the K-means algorithm that determines the membership values given the mean distances between elements.

There are multiple definitions of fuzzy partition according to the application, most of these definitions are posed in the universe of fuzzy sets, so that observing the relationship between fuzzy sets and the algebra $\mathcal{K}_{3}$, the possibility of formulating an alternative way from the point of view of this algebra becomes evident, in order to make use of its properties, this will be denoted as $\mathcal{A}$-partition.

An $\mathcal{A}$-partition is a definition formulated from the three-valued $\mathcal{K}_{3}$ Kleene algebra and has appropriate characteristics for a fuzzy clustering as the C-means algorithm and it is expected that by handling a finite set of states, a fuzzy set with better interpretability will be obtained because a finite set can simplify the definition of linguistic labels.

Initially some theoretical concepts and examples related to partitions and finite algebras are described,we are going to have a look through the different definitions proposed in the literature; finite algebras particularly the $\mathcal{K}_{3}$-algebra; well-formed formulas and regularity; then the homomorphism between fuzzy sets and the algebra described; the crossing set is defined and based on this, the $\mathcal{A}$-partition is proposed; reduction methods are used on the proposed definition and then the clustering algorithm is designed (using the $\mathrm{C}$-means as as a reference framework) applying a homomorphism and $\mathcal{A}$-partition detailing its steps and a corresponding experimentation, comparison, discussion and conclusions are made. 


\section{Background information}

The partition element definitions are an extension of the crisp definition where two important concepts are required, covering (the union of a family of subsets forms the totality of the set to which they belong) and disjoint sets (two sets have no elements in common). Over time the paradigm of sets has developed from the crisp set theory from which the concept of crisp partition is derived. [2] [3] [4.

A family $\left\{A_{i}: i \in I\right\}$ of non-empty classical subsets of $A$ is a partition if and only if

- $A_{i} \cap A_{j}=\varnothing$ with $i \neq j\left(A_{i}\right.$ and $A_{j}$ are disjoint $)$

- $\bigcup_{i \in I} A_{i}=A$ (partition elements have covering over the whole set $A$ ).

With the advent of fuzzy set theory proposed by Zadeh [5] has given rise to several definitions of partition element The first definition is attributed to Ruspini[1] and it is done based on the membership functions of the fuzzy sets of a universe $X$. This definition is as follows.

A finite family $\left\{A_{i}: i \in I\right\}$ of nonempty fuzzy subsets of the universal set $X$ is a partition if and only if for all $x \in X$ is satisfied.

$$
\sum_{i \in I} \mu_{A_{i}}(x)=1
$$

where $\mu_{A_{i}}(x)$ is the membership function of the subset $A_{i}$.

Bezdek and Harris [6], Dumitrescu 7 and Ovchinnikov 8 applied the fuzzy sets operations. For this purpose they expressed the intersection as a t-norm $\triangle$ and the union as its respective dual t-conorm $\nabla$ defined as:

$$
x \nabla y=1-(1-x) \triangle(1-y) \text { for } x, y \in[0,1] .
$$

A finite Family $\left\{A_{i}: i \in I\right\}$ of non-empty fuzzy subsets of the universal set $X$ is a partition if and only if, for all $x \in X$, the following is true

- $\mu_{A_{i}}(x) \triangle \mu_{A_{j}}(x)=0$ with $i \neq j\left(A_{i}\right.$ and $A_{j}$ are disjoint $)$

- $\underset{i \in I}{\nabla} \mu_{A_{i}}(x)=1$ (the partition elements have coverage over the entire universal set).

An alternative way is the definition given by Butnariu $[9]$ who modifies the meaning of disjoint sets.

A finite family $\left\{A_{i}: i \in I\right\}$ of non-empty fuzzy subsets of the universal set $X$ is a partition if and only if, for all $x \in X$, the following is true

- $\left(\nabla_{i=1}^{j} \mu_{A_{i}}(x)\right) \triangle \mu_{A_{j+1}}(x)=0$ with $j=1,2, \ldots n-1\left(\left\{A_{i}: i \in I\right\}\right.$ are disjoint $)$

- $\underset{i \in I}{\nabla} \mu_{A_{i}}(x)=1$ (the partition elements have coverage over the entire universal set).

Markechová 10]. A finite family $\left\{\tilde{A}_{i}: i \in I\right\}$ of non-empty fuzzy subsets of the universal set $X$ is called a fuzzy w-partition 1 if and only if, for all $x \in X$, the following is satisfied

- $\left(\bigvee_{i \in I} \mu_{A_{i}}(x)\right)^{\prime} \leq \bigvee_{i \in I} \mu_{A_{i}}(x)$

- $\left(\mu_{A_{i}}(x) \wedge \mu_{A_{j}}(x)\right) \leq\left(\mu_{A_{i}}(x) \wedge \mu_{A_{j}}(x)\right)^{\prime}$

\footnotetext{
${ }^{1}$ Also called weak fuzzy partition
} 
where $\vee, \wedge$ and ' are the minimum t-norm, the maximum t-norm and the standard complement. Chakraborty and Das [1] proposed another definition of fuzzy partition based on the concept of disjoint set.

A family $\left\{A_{i}: i \in I\right\}$ of non-empty fuzzy subsets of the set $A$ is a fuzzy partition of $A$ if and only if

- $A_{i} \cap A_{j}=\varnothing$ with $i \neq j\left(A_{i}\right.$ and $A_{j}$ are disjoint $)$

- $\bigcup_{i \in I} A_{i}=A$ (the partition elements have coverage over the entire set $A$.)

where $\cap$ and $\cup$ are defined as the infimum and supremum, respectively.

Bhakat and Das [12]. A family $\left\{A_{i}: i \in I\right\}$ of nonempty fuzzy subsets of the set $A \in X$ is a q-fuzzy partition of $A$ if and only if, for all $x \in X$, it is satisfied that

- $\mu_{A_{i}}(x)+\mu_{A_{j}}(x) \leq 1$, for $i \neq j\left(A_{i}\right.$ and $A_{j}$ are disjoint sets $)$

- $\bigcup_{i \in I} A_{i}=A$ (the partition elements have coverage over the set $A$ )

Montes, et al. introduced the concept of $\delta$ - $\varepsilon$-partition by making use of the concept of $\alpha$-cut (noted as ${ }^{\alpha} A$ ) of the set $A$.

A family of fuzzy sets $\left\{A_{i}: i \in I\right\}$ is a $\delta$ - $\varepsilon$-fuzzy partition of $A$, with $0 \leq \varepsilon<\delta \leq 1$ if and only if

- ${ }^{\alpha}\left(A_{i} \cap A_{j}\right)=\varnothing$ with $i \neq j\left(A_{i}\right.$ and $A_{j}$ are disjoint sets)

- ${ }^{\alpha}\left(\bigcup_{i \in I} A_{i}\right)={ }^{\alpha} A$ (the partition elements $A_{i}$ have coverage over the whole set $\left.{ }^{\alpha} A\right)$

for all $\alpha \in(\varepsilon, \delta)$, where $\cap$ and $\cup$ are described by any t-norm and any t-conorm, respectively. Let " $=\varepsilon "$ an equality in terms of alpha cuts such that:

$$
A_{1}={ }_{\varepsilon} A_{2} \Longleftrightarrow{ }^{\alpha} A_{1}={ }^{\alpha} A_{2} \forall \alpha \in\left(\varepsilon, \varepsilon^{\prime}\right)
$$

A family of fuzzy sets $\left\{A_{i}: i \in I\right\}$ is a fuzzy $\varepsilon$-partition of $A$, with $0 \leq \varepsilon \leq 0.5$ if and only if

- $A_{i} \cap A_{j}={ }_{\varepsilon} \varnothing$ with $i \neq j\left(A_{i}\right.$ and $A_{j}$ are disjoint sets)

- $\bigcup_{i \in I} A_{i}={ }_{\varepsilon} A$ (the partition elements $A_{i}$ have coverage over the whole set ${ }^{\varepsilon} A$ )

where $\cap$ and $\cup$ are described by any t-norm and any t-conorm, respectively. In 13] we extend the definition of $\delta$ - $\varepsilon$-partition for finite interval fuzzy sets.

These partition definitions have a restriction due to their covering, which limits the transition between partition elements in a fuzzy way, since it prevents the existence of regions in which not

necessarily $\bigcup_{i \in I} A_{i}=A$, which implies that for all $x$ there must exist at least one partition element whose membership function that is equal to the set to be partitioned.

\section{Preliminaries}

Definition 1 (Homomorphism) [14 [15] Given two algebraic structures $\mathcal{A}=\left(A, \circ_{1}, \ldots, \circ_{k}\right)$ and $\mathcal{B}=\left(B, *_{1}, \ldots, *_{k}\right)$ of the same type, a function $T$ between them

$$
T: \mathcal{A} \rightarrow \mathcal{B}
$$

is said homomorphism if and only if:

$$
T\left(\circ\left(a_{1}, \ldots, a_{n}\right)\right)=*_{i}\left(T\left(a_{1}\right), \ldots, T\left(a_{n}\right)\right)
$$

For all $i=1, \ldots, k$ and $a_{1}, \ldots a_{n} \in A$ 
One of the most important concepts in fuzzy set theory is the concept of $\alpha$-cut which basically allows to make a horizontal cut over the fuzzy sets and is defined as:

Definition 2 ( $\alpha$-cut and strong $\alpha$-cut) [2] 3] Given a fuzzy set $A$ defined on $X$ and any number $\alpha \in[0,1]$ the $\alpha$-cut ${ }^{\alpha} A$ and the strong $\alpha$-cut ${ }^{\alpha+} A$ are the classic sets:

$$
\begin{gathered}
{ }^{\alpha} A=\left\{x \mid \mu_{A}(x) \geq \alpha\right\} \\
{ }^{\alpha+} A=\left\{x \mid \mu_{A}(x)>\alpha\right\}
\end{gathered}
$$

Example 3.1 the figure 3.1 shows the Gaussian fuzzy set $A$ from the universe $X$ and two $\alpha$-cut over that set, $\alpha_{1}$ and $\alpha_{2}$ generating the sets ${ }^{\alpha_{1}} A$ and ${ }^{\alpha_{2}} A$.

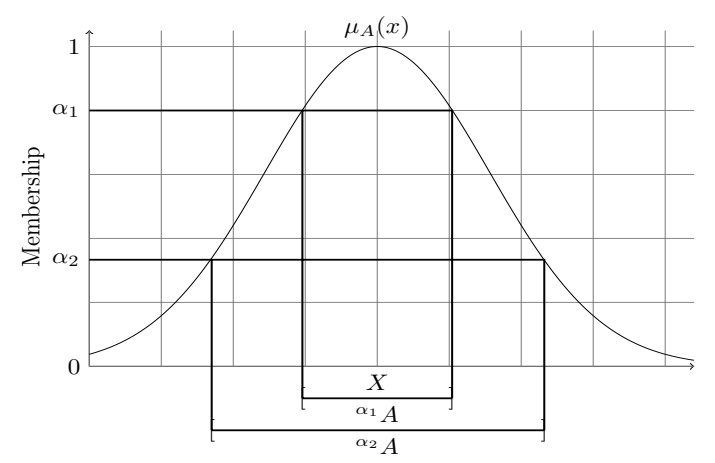

Figure 3.1. Gaussian fuzzy set $A$ and its alpha cuts $\alpha_{1}$ and $\alpha_{2}$

Definition 3 (Fuzzy partition proposed by Ruspini) [1] A finite first-order fuzzy partition of a set $X$ is a finite family of fuzzy subsets $\left\{A_{1}, A_{2}, \ldots, A_{n}\right\}$ of the universe $X$ that satisfy the condition

$$
\sum_{i=1}^{n} \mu_{i}(x)=1 \quad \text { for } \quad \text { all } \quad x \in U .
$$

Definition 4 (Lattice) [2] $[14][16][17]$

A lattice $\mathcal{L}=\langle L ; \leq\rangle$ is a partially ordered set in which, for every pair of elements $a, b \in L$, there exists a supremum element $\sup \{a, b\}$ and an infimum element $\inf \{a, b\}$. In other words, $\mathcal{L}$ is a lattice if $\sup \{H\}$ and $\inf \{H\}$ exist for every subset $H$ of $L$.

The supreme element $\sup \{a, b\}$ and the smallest element $\inf \{a, b\}$ are usually denoted by the logical operation disjunction $a \vee b$ and conjunction $a \wedge b$ respectively and satisfy the following properties for all $a, b \in L$ :

$$
\begin{aligned}
a \vee a & =a \\
a \wedge a & =a \\
a \vee a & =b \vee a \\
a \wedge b & =b \wedge a \\
(a \vee b) \vee c & =a \vee(b \vee c) \\
(a \wedge b) \wedge c & =a \wedge(b \wedge c) \\
a \vee(a \wedge b) & =a \\
a \wedge(a \vee b) & =a
\end{aligned}
$$

Two other properties relating $\vee$ and $\wedge$ with the partial order relationship $\leq$ are:

$$
\begin{aligned}
& a \leq b \Leftrightarrow a \vee b=b \\
& a \leq b \Leftrightarrow a \wedge b=a
\end{aligned}
$$


All reticule $\mathcal{L}$ satisfies the distributivity inequalities for all $a, b, c \in A$ given by:

$$
\begin{aligned}
& a \vee(b \wedge c) \geq(a \vee b) \wedge(a \vee c) \\
& a \wedge(b \vee c) \leq(a \wedge b) \vee(a \wedge c)
\end{aligned}
$$

Definition 5 (Distributive lattice ) A Distributive lattice $\mathcal{L}=\langle L ; \leq\rangle$ is a lattice satisfying the distributivity properties for binary operations $\vee$ and $\wedge$ for all $a, b, c \in L$.

$$
\begin{aligned}
& a \vee(b \wedge c)=(a \vee b) \wedge(a \vee c) \\
& a \wedge(b \vee c)=(a \wedge b) \vee(a \wedge c)
\end{aligned}
$$

Definition 6 (bounded lattice) $A$ bounded lattice $\mathcal{L}=\langle L ; \leq\rangle$ is the one where the elements zero and unity exist in $L$, denoted by 0 and 1 , such that for every element $a \in L$ the identity properties are satisfied for the operations $\vee$ and $\wedge$.

$$
\begin{aligned}
& a \vee 0=a \\
& a \wedge 1=a
\end{aligned}
$$

From the equation (3.6) it follows that 1 is the maximum element of $L$ and the element 0 is the minimum element. Both are unique in $L$. From the property of identity and the property of absorption we deduce the property of absorption by 1 and 0 .

$$
\begin{aligned}
& a \vee 1=1 \\
& a \wedge 0=0
\end{aligned}
$$

Definition 7 (Complemented lattice) A complemented lattice $\mathcal{L}=\langle L ; \leq\rangle$ is a bounded lattice, in which for every element $a \in L$ there is an element $a^{\prime} \in L$ called the complement of a such that the complementation properties are satisfied.

$$
\begin{aligned}
& a \vee a^{\prime}=1 \\
& a \wedge a^{\prime}=0
\end{aligned}
$$

The De Morgan, Kleene and Boolean algebras are defined below. These are lattice structures and are therefore governed by the standard t-norm, t-conorm and complement operations. You can consult [2][4][18][19] for a more detailed study.

Definition 8 (De Morgan Algebra) A De Morgan Algebra $\mathcal{M}$ is a algebra $\langle A ; \vee, \wedge$, $', 0,1\rangle$, where $\langle A ; \vee, \wedge, 0,1\rangle$ is a bounded distributive lattice. That is, it satisfies the properties (3.1)(3.7). Binary disjunction operations $(\vee)$ and conjuntion $(\wedge)$ together with the unary involute complement operation (' ) satisfy the property of involution and De Morgan's laws for all $a, b \in A$.

$$
\begin{aligned}
\left(a^{\prime}\right)^{\prime} & =a \\
(a \vee b)^{\prime} & =a^{\prime} \wedge b^{\prime} \\
(a \wedge a)^{\prime} & =a^{\prime} \vee b^{\prime}
\end{aligned}
$$

Definition 9 (Kleene's algebra) A Kleene's algebra $\mathcal{K}=\left\langle A ; \vee, \wedge,{ }^{\prime}, 0,1\right\rangle$ is a De Morgan algebra that satisfies the following inequality for all $a, b \in A$ :

$$
a \wedge b^{\prime} \leq b \vee b^{\prime}
$$

This inequality can also be written as $\left(a \wedge a^{\prime}\right) \wedge\left(b \vee b^{\prime}\right)=a \wedge a^{\prime}$ or as $\left(a \wedge a^{\prime}\right) \vee\left(b \vee b^{\prime}\right)=b \vee b^{\prime}$. These equations are also called Kleene's laws. 20]. 
Definition 10 (Boolean algebra) $A$ Boolean algebra $\mathcal{B}$ is a Kleene algebra that satisfies the complementation properties for all $a, b \in A$.

$$
\begin{aligned}
& a \vee a^{\prime}=1 \\
& a \wedge a^{\prime}=0
\end{aligned}
$$

\section{Definition 11 (Three-valued algebra)}

An algebra $\mathcal{K}_{3}=\left\langle K_{3} ; \vee, \wedge,{ }^{\prime}, 0,1\right\rangle$, with $K_{3}=\{0, u, 1\}$, is a three-element algebra where $\left\langle K_{3} ; \vee, \wedge, 0,1\right\rangle$ is the lattice shown in the figure 3.2 .

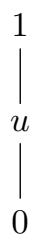

Figure 3.2. Lattice diagram of the algebra $\mathcal{K}_{3}$.

$\mathcal{K}_{3}$ is a set of three elements satisfying the total order relation $0<u<1$. The conjunction, disjunction and complement operations are described in the table $3.1 . \mathcal{K}_{3}$ is a finite Kleene algebra. [2] 4] 21] [19] [22]. Therefore, it fulfills the properties (3.1)-(3.11). It does not satisfy the property (3.12) since $u \vee u^{\prime}=u \vee u=u \neq 1$ and $u \wedge u^{\prime}=u \wedge u=u \neq 0$.

Table 3.1. Tables of operations in $\mathcal{K}_{3}$

(a) Disjunction, (b) conjunction and (c) complement.

\begin{tabular}{c|ccc}
$\vee$ & 0 & $u$ & 1 \\
\hline 0 & 0 & $u$ & 1 \\
$u$ & $u$ & $u$ & 1 \\
1 & 1 & 1 & 1
\end{tabular}

\begin{tabular}{l|lll}
$\wedge$ & 0 & $u$ & 1 \\
\hline 0 & 0 & 0 & 0 \\
$u$ & 0 & $u$ & $u$ \\
1 & 0 & $u$ & 1
\end{tabular}

\begin{tabular}{c|c}
$a$ & $a^{\prime}$ \\
\hline 0 & 1 \\
$u$ & $u$ \\
1 & 0
\end{tabular}

A formula is a concatenation of constants, variables, symbols and operators that obey certain rules to make sense.

Definition 12 (Well-formed set of formulas $F$ ) [4] [23] 24$]$ Let $V=\{x, y, z, \ldots\}$ be a finite set of variables. $F$ is the smallest set of concatenations of the constants 0 and 1 , the elements of $V$ and and the operation symbols $\vee, \wedge$ and' that satisfies

1. $0 \in F, 1 \in F$ and if $x \in V$ then $x \in F$.

2. If $f, g \in F$ then $f \vee g \in F, f \wedge g \in F$ and $f^{\prime} \in F$

The elements of the set $F$ are called well-formed formulas or simply formulas.

The first item determines the initial elements of the set $F$, from which it follows that $V \cup\{0,1\} \subseteq F$. The second item determines that known formulas can be concatenated to construct new formulas, making use of the operation symbols $\vee, \wedge$ and '. $F$ is an infinite set [4].

In $F$ the value of formulas is not set, that is, different strings have no connected meaning. For instance the strings $x \vee y$ and $y \vee x$ are different. The value of the formulas is introduced by an value algebra $\mathcal{A}$. Some value algebras can be $\mathcal{L} \mathcal{M}, \mathcal{K}, \mathcal{B}, \mathcal{K}_{3}$ and $\mathcal{B}_{2}$.

The concept of regularity is an important aspect for the treatment of tables in $\mathcal{K}_{3}$ algebra when we want to obtain formulas from them and in this paper it is a useful tool for the determination of partitions based on $\mathcal{K}_{3}$.

Definition 13 [25] Let the set $V=\{0, u, 1\}$. Let $\propto$ be a partial order relation such that $0 \propto u$, $1 \propto u$ and $a \propto a, a \in V, 0$ and 1 are not comparable to each other. This relation can be extended to $V^{n}$. For two elements $A=\left(a_{1}, \ldots, a_{n}\right)$ and $B=\left(b_{1}, \ldots, b_{n}\right)$ of $V^{n}, A \propto B$ if and only if $b_{i} \propto a_{i}$ for all values of $i$ and is said $B$ to be less or equally ambiguous than $A$. 
Definition 14 (Regularity) [25] A function $F$ is called regular if it fulfill the following conditions:

- Condition 1: if $F$ is a ternary function, that is, it can be represented by a well-formed formula.

- Condition 2: if $F(A) \in 0,1$, then $F(B)=F(A)$ for all $B$ that $B \propto A 2^{2}$

- Condition 3: if $B \propto A$ then $F(B) \propto F(A)$, it is called a ternary logic function.

In 25 it is proved that the three conditions are equivalent. By considering two algebras: $\left\langle\mathcal{F}(X), \cup, \cap,{ }^{\prime}\right\rangle$ the fuzzy sets and their standard operations and $\left\langle\mathcal{K}_{3}, \wedge, \vee,{ }^{\prime}\right\rangle$ the algebra and its conjunction, disjunction and complement operations, there is a function $f$

$$
f: \mathcal{F}(X) \rightarrow \mathcal{K}_{3}
$$

which is a homomorphism for each of its operations. This function is stated in 27$][4$ given the similarities in their structure and properties.

This homomorphism $f$ is described as a mapping of the values $\{0, u, 1\}$ to the membership levels of the fuzzy sets.

The function $f$ is determined by the following encoding:

Trivalent coding of operating regions: $[27]$ given a $\varepsilon \in[0,1]$ defined by the designer, such that $0<\varepsilon<\zeta$, where $\zeta$ is the fixed point of the logical operation "no" (or complement $\left(^{\prime}\right)$ ). Each fuzzy set $A$ performs a partitioning of the input universe $X$ in the following way:

a Coding with 0: for those $x \in X$ tales que $\mu_{A}(x) \in[0, \varepsilon)$

b Coding with $\mathbf{u}$ : for those $x \in X$ such that $\mu_{A}\left(x_{i}\right) \in\left[\varepsilon, \varepsilon^{\prime}\right]$

c Coding with 1:for those $x \in X$ such that $\mu_{A}(x) \in(\varepsilon, 1]$

In this step it is possible to perform a trivalent encoding of all possible regions of operation by means of a trivalent logic truth table. $(0, u, 1)$, based on the division described above. Each coding will correspond to a row in the table.

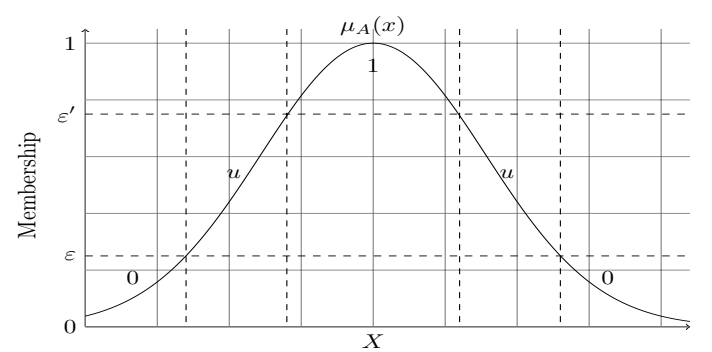

Figure 3.3. Assigning Kleenean values to a fuzzy function

\section{Development}

Studying several definitions of fuzzy partition, present in the literature, a common pattern is found in the determination of the properties of covering of sets, where the union operation is used, and disjunct sets (or disjunctivity), which makes use of the intersection operation. We seek to propose such properties in the 3 -element Kleenean algebra $\mathcal{K}_{3}$, in which the conjunction and disjunction operations are analogous to the standard intersection and union operations of fuzzy sets given the homomorphism T. To achieve this goal, the following concept is proposed which will be used to determine the value of the variables in $\mathcal{K}_{3}$ that are the product of the mapping of the evaluated fuzzy sets into the regions where two or more membership functions present in the interval $\left[\varepsilon, \varepsilon^{\prime}\right]$ intersect.

\footnotetext{
${ }^{2}$ This condition is an extension of the definition present in 26
} 


\subsection{Crossing set}

Definition 15 (Crossing set) Let there be two non-empty fuzzy sets $A_{i}$ and $A_{j}$ (with $i \neq j$ ) subsets of the universal set $X$. Let $\mathcal{K}_{i j}$, a subset of the universal set $X$, be defined as follows:

$$
\begin{array}{r}
\aleph_{i j}=\left\{x \mid \varepsilon \leq \mu_{A_{i}}(x) \leq \varepsilon^{\prime} \quad\right. \text { and } \\
\left.\varepsilon \leq \mu_{A_{j}}(x) \leq \varepsilon^{\prime}\right\} .
\end{array}
$$

$\mathrm{K}_{i j}$ is called a crossing set and $A_{i}$ and $A_{j}$ are called neighboring sets if $\aleph_{i j} \neq \varnothing$.

Example 4.1 Let the sets $A_{1}, A_{2}$ and $A_{3}$ be subsets of the universal set $X$, with their respective membership functions $\mu_{A_{1}}(x), \mu_{A_{2}}(x)$ and $\mu_{A_{3}}(x)$ shown in the figure 4.1 . The crossing sets $K_{12}$ and $K_{23}$ are defined as the elements of the universe $X$ belonging to the shaded regions, as indicated by the indicator functions $\mu_{K_{12}}(x)$ and $\mu_{K_{23}}(x)$. Since the sets $K_{12}$ and $\mathcal{K}_{23}$ are non-empty, it is said that $A_{1}$ is a neighbor of $A_{2}$ and $A_{2}$ in turn is a neighbor of(no estoy seguro de esta frase) $A_{3}$. The set $\mathcal{K}_{13}$ is empty, therefore $A_{1}$ and $A_{3}$ are not neighbors

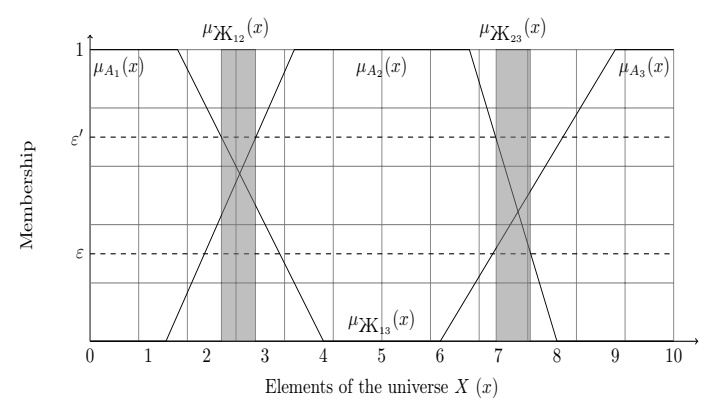

Figure 4.1. Crossing sets for the example 4.1

The definition 4.1 allows one to identify the regions of the universe $X$ where sets map to $u$ according to the algebra $\mathcal{K}_{3}$. These regions can be expressed in terms of the alpha-cuts and strong alpha-cuts of the membership functions in addition to which the above regions can be employed to determine whether a family of sets is a partition. The above properties are stated in the following two propositions.

Proposition 1 The crossing set can be described as:

$$
\mathrm{K}_{i j}={ }^{\varepsilon} A_{i} \cap \overline{\left(\varepsilon^{\prime}\right)^{+} A_{i}} \cap{ }^{\varepsilon} A_{j} \cap \overline{\left(\varepsilon^{\prime}\right)^{+} A_{j}}
$$

Proof. The alpha cuts of the set $A_{i}$ are defined as follows:

$$
\begin{aligned}
& { }^{\varepsilon} A_{i}=\left\{x \mid \varepsilon \leq \mu_{A_{i}}(x)\right\} \\
& { }^{(1-\varepsilon)^{+}} A_{i}=\left\{x \mid \varepsilon^{\prime}<\mu_{A_{i}}(x)\right\}
\end{aligned}
$$

The definition is similar for $A_{j}$.

By rewriting equation 4.2 using the above expressions and making use of the definition of the intersection operation between sets, the following results

$$
\begin{array}{r}
{ }^{\varepsilon} A_{i} \cap \overline{\left(\varepsilon^{\prime}\right)^{+} A_{i}} \cap{ }^{\varepsilon} A_{j} \cap \overline{\left(\varepsilon^{\prime}\right)^{+} A_{j}}=\left\{x \mid \varepsilon \leq \mu_{A_{i}}(x) \quad\right. \text { and } \\
\varepsilon^{\prime} \geq \mu_{A_{i}}(x) \text { and } \\
\varepsilon \leq \mu_{A_{j}}(x) \text { and } \\
\left.\varepsilon^{\prime} \geq \mu_{A_{j}}(x)\right\} \\
{ }^{\varepsilon} A_{i} \cap \overline{\left(\varepsilon^{\prime}\right)^{+} A_{i}} \cap{ }^{\varepsilon} A_{j} \cap \overline{\left(\varepsilon^{\prime}\right)+A_{j}}=\left\{x \mid \varepsilon \leq \mu_{A_{i}}(x) \leq \varepsilon^{\prime} \quad\right. \text { and } \\
\left.\varepsilon \leq \mu_{A_{j}}(x) \leq \varepsilon^{\prime}\right\} .
\end{array}
$$


This shows that the expressions 4.1 and 4.2 are equivalent

Example 4.2 Let $A_{1}, A_{2}$ and $A_{3}$ be the sets of example 4.1 . Figure 4.2 shows how the crossing sets are constructed by means of the alpha cuts, for which we make use is made of their indicator functions. According to expression 4.2 the crossing sets are defined as follows

- $\mathrm{K}_{12}={ }^{\varepsilon} A_{1} \cap \overline{\left(\varepsilon^{\prime}\right)^{+} A_{1}} \cap{ }^{\varepsilon} A_{2} \cap \overline{\left(\varepsilon^{\prime}\right)^{+} A_{2}}$

- $K_{23}={ }^{\varepsilon} A_{2} \cap \overline{\left(\varepsilon^{\prime}\right)^{+} A_{2}} \cap{ }^{\varepsilon} A_{3} \cap \overline{\left(\varepsilon^{\prime}\right)^{+} A_{3}}$

Figures $4.2 \mathrm{~b}$ and $4.2 \mathrm{c}$ show that the crossing set $\mathrm{K}_{12}$ can be reduced to the expression $\mathrm{K}_{12}=$ $\overline{\left(\varepsilon^{\prime}\right)^{+} A_{1}} \cap \overline{\left(\varepsilon^{\prime}\right)^{+} A_{2}}$; in turn, figures $4.2 \mathrm{a}$ and $4.2 \mathrm{c}$ allow us to visualize that the crossing set $\mathcal{K}_{23}$ can be reduced to the expression $K_{23}={ }^{\varepsilon} A_{2} \cap{ }^{\varepsilon} A_{3}$

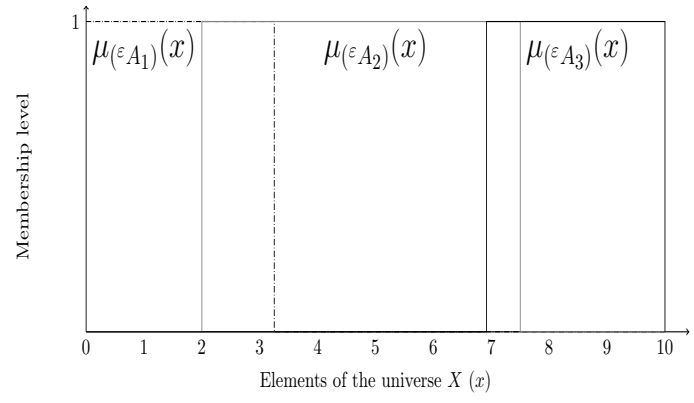

(a) Alpha cuts

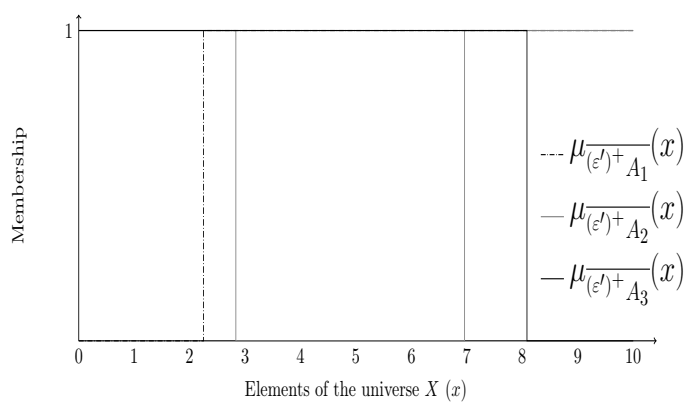

(b) Alpha cut complements

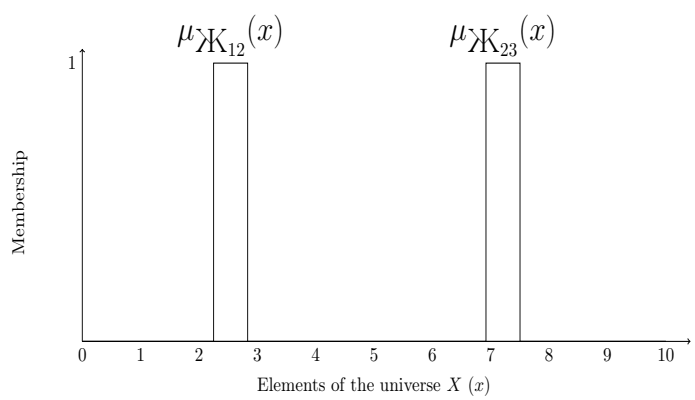

(c) Crossing sets

Figure 4.2. Representation of the crossing sets of the example 4.2 by alpha cuts.

$K_{T}$ is the union of all possible crossing sets $\left\{K_{i j}: i, j \in I\right\}$ with $i \neq j$ formed by a family of fuzzy sets $\left\{A_{i}: i \in I\right\}$. In this way

$$
\mathrm{K}_{T}=\bigcup_{i, j \in I} \mathrm{~K}_{i j} \text { with } \mathrm{i} \neq \mathrm{j}
$$

The homomorphism $T: \mathcal{F}(X) \rightarrow \mathcal{K}_{3}$ between fuzzy sets and the three-valued Kleene algebra applied to a fuzzy set $A$ is expressed as follows

$$
T\left(\mu_{A}(x)\right)=a
$$

Taking into account the above notions, we propose a definition that seeks to satisfy the classical and fuzzy behaviors considering the sets $K_{i j}$.

\section{Definition 16 (A-partition)}

Let be a fuzzy set $A$ subset of the universal set $X$ and $\Pi=\left\{A_{i}: i \in I\right\}$ a finite family of nonempty subsets of $A$; let $\mathcal{K}_{T}$ be the union of all nonempty crossing sets generated by $\Pi$; let $T: \mathcal{F}(X) \rightarrow \mathcal{K}_{3}$ the homomorphism 4.3 such that $T\left(\mu_{A_{i}}(x)\right)=a_{i} \forall i \in I$. II is said to be a fuzzy partition if and only if it is satisfied, $\forall x \in X$ : 
Covering (A-covering):

$$
d=\bigvee_{i \in I} a_{i}=\left\{\begin{array}{cc}
a & \forall x \in \overline{\mathcal{K}_{T}} \\
u & \forall x \in \mathcal{K}_{T}
\end{array}\right.
$$

Disjoint sets (A-disjunctiveness):

$d_{c}=\bigvee_{j=1}^{n-1} \bigvee_{i=j+1}^{n}\left(a_{j} \wedge a_{i}\right)= \begin{cases}0 & \forall x \in \overline{K_{T}} \\ u & \forall x \in \mathcal{K}_{T}\end{cases}$

where $n$ is the number of elements of $\Pi$.

The property 4.4 indicates that the disjunction of the variables associated to all the elements of the family $\Pi$ is equal to the variable associated to the set $A\left(a \in \mathcal{K}_{3}\right)$ for the elements that belong to $\overline{K_{T}}$ and is equal to $u$ for the elements belonging to $\aleph_{T}$. In turn, the property 4.5 indicates that the disjunction of all possible conjunctions of two different variables associated with elements of family $\Pi$ is equal to 0 for elements belonging to $\overline{K_{T}}$ and is equal to $u$ for elements belonging to $K_{T}$. In the definition 16 it is evident that, both in the covering and in the disjoint sets, the disjunction and conjunction of the variables of $\mathcal{K}_{3}$ is equal to $u$ for the elements belonging to the non-empty crossing sets.

However, the proposed definition contains both kleenean variables $\left(a,\left\{a_{i}: i \in I\right\}\right)$ and classical sets (crossing sets $K$ ). We want to find an equivalence with purely algebraic terms and for this purpose we look for a way to determine crossing sets through kleenean variables coming from the homomorphism 4.3, using $d, d_{c}, a$ and the kleenan properties of these variables with which it is desired to pose the conditions equivalent to the definition 16 in terms of tables and formulas. When $d$ and $d_{c}$ are equal to $u$, they guarantee the existence of non-empty crossing sets, since they imply the existence of two or more sets mapping to that value for the same elements.

To observe the elements that meet the conditions of covering and disjoint sets, these are restricted to the values that can be presented in a truth table. Since $\left\{A_{i}: i \in I\right\}$ is a subset of $A$ it must be satisfied $d \leq a$ and it is evident $d_{c} \leq d$ so values that do not satisfy these conditions are discarded. Finally the inequality must be satisfied:

$$
d_{c} \leq d \leq a
$$

The variables $d$ and $d_{c}$ describe tables, these together with the inequality (4.6), which eliminates unnecessary combinations, can be used to determine the conditions for the 4.4 and 4.5 as shown in the table 4.1. According to the membership to a crossing set, the covering condition and disjoint sets are determined. The elements that satisfy both conditions are considered a $\mathcal{A}$-partition.

Table 4.1. Definition's table of the $\mathcal{A}$-partition properties. This table shows the possible combinations taking into account the restrictions of the inequality 4.6 in the columns $\mathcal{K}, \mathcal{A}$-covering, $\mathcal{A}$-disjointness and $\mathcal{A}$-partition $\checkmark$ indicates that the conditions are met, and $\boldsymbol{x}$ indicates that the conditions are not met.

\begin{tabular}{ccc|cccc}
$a$ & $d$ & $d_{c}$ & X & $\mathcal{A}$-covering & $\mathcal{A}$-disjointness & $\mathcal{A}$-partition \\
\hline 0 & 0 & 0 & $\boldsymbol{X}$ & $\checkmark$ & $\checkmark$ & $\checkmark$ \\
$u$ & 0 & 0 & $\boldsymbol{x}$ & $\boldsymbol{x}$ & $\checkmark$ & $\boldsymbol{x}$ \\
$u$ & $u$ & 0 & $\boldsymbol{x}$ & $\checkmark$ & $\checkmark$ & $\checkmark$ \\
$u$ & $u$ & $u$ & $\checkmark$ & $\checkmark$ & $\checkmark$ & $\checkmark$ \\
1 & 0 & 0 & $\boldsymbol{x}$ & $\boldsymbol{x}$ & $\checkmark$ & $\boldsymbol{x}$ \\
1 & $u$ & 0 & $\boldsymbol{x}$ & $\boldsymbol{x}$ & $\checkmark$ & $\boldsymbol{x}$ \\
1 & $u$ & $u$ & $\checkmark$ & $\checkmark$ & $\checkmark$ & $\checkmark$ \\
1 & 1 & 0 & $\boldsymbol{x}$ & $\checkmark$ & $\checkmark$ & $\boldsymbol{x}$ \\
1 & 1 & $u$ & $\boldsymbol{x}$ & $\boldsymbol{x}$ & $\boldsymbol{x}$ & $\boldsymbol{x}$ \\
1 & 1 & 1 & $\boldsymbol{x}$ & $\checkmark$ & $\boldsymbol{x}$ &
\end{tabular}

The conditions of the definition 16 can be rewritten through an equality taking into account the relation of the elements involved, since they can be interpreted as Kleenean truth tables and in turn these can be seen as formulas in case they are regular. 
Proposition 2 Let a fuzzy set $A$ be a subset of the universal set $X$ and a finite family $\Pi=\left\{A_{i}\right.$ : $i \in I\}$ of nonempty subsets of $A$; let $T: \mathcal{F}(X) \rightarrow \mathcal{K}_{3}$ be the homomorphism 4.3. $\Pi$ is said to be a fuzzy $\mathcal{A}$-partition if and only if it is satisfied, $\forall x \in X$ :

$$
d=a \wedge d_{c}^{\prime}
$$

For all $d_{c}$, $d$ and a satisfying inequality 4.6 .

Proof. Table 4.2 shows the truth values of $a, d$ and $d_{c}$ where inequality 4.6 . If we take the values of the rows in which we have an $\mathcal{A}$-partition $(\checkmark)$ and with them we establish the disjunctive normal form for $a$ and $d_{c}$, setting as output the variable $d$, ignoring the other combinations, we obtain

$$
d=\left(a \wedge a^{\prime} \wedge d_{c}{ }^{\prime}\right) \vee\left(a \wedge a^{\prime} \wedge d_{c} \wedge d_{c}{ }^{\prime}\right) \vee\left(a \wedge d_{c} \wedge d_{c}{ }^{\prime}\right) \vee\left(a \wedge d_{c}{ }^{\prime}\right) .
$$

Using some properties of the $\mathcal{K}_{3}$ algebra the simplification of the above formula is performed.

$$
\begin{gathered}
d=\left(\left(a \wedge a^{\prime}\right) \vee\left(a \wedge a^{\prime} \wedge d_{c}\right) \vee\left(a \wedge d_{c}\right) \vee a\right) \wedge d_{c}{ }^{\prime} \\
d=\left(\left(a \wedge a^{\prime}\right) \vee a\right) \wedge d_{c}{ }^{\prime} \\
d=a \wedge d_{c}{ }^{\prime}
\end{gathered}
$$

This gives the formula 4.7

Table 4.2. Determination of the disjunctive normal form (DNF) for $d$ respectively to $a$ and $d_{c}$ for values satisfying inequality 4.6

\begin{tabular}{cc|cc|c}
$a$ & $d_{c}$ & $d$ & $\mathcal{A}$-partition & $D N F$ \\
\hline 0 & 0 & 0 & $\checkmark$ & \\
$u$ & 0 & 0 & $\boldsymbol{X}$ & \\
$u$ & 0 & $u$ & $\checkmark$ & $a \wedge a^{\prime} \wedge d_{c}{ }^{\prime}$ \\
$u$ & $u$ & $u$ & $\checkmark$ & $a \wedge a^{\prime} \wedge d_{c} \wedge d_{c}{ }^{\prime}$ \\
1 & 0 & 0 & $\boldsymbol{X}$ & \\
1 & 0 & $u$ & $\boldsymbol{X}$ & \\
1 & $u$ & $u$ & $\checkmark$ & $a \wedge d_{c} \wedge d_{c}{ }^{\prime}$ \\
1 & 0 & 1 & $\checkmark$ & $a \wedge d_{c}{ }^{\prime}$ \\
1 & $u$ & 1 & $\boldsymbol{X}$ & \\
1 & 1 & 1 & $\boldsymbol{X}$ &
\end{tabular}

When the set $A$ is the universal set, the variable $a$ takes the value of 1 ; then the condition 4.7 is reduced to

$$
\begin{gathered}
d=d_{c}{ }^{\prime} \wedge a=d_{c}{ }^{\prime} \wedge 1 \\
d=d_{c}{ }^{\prime}
\end{gathered}
$$

The formula 4.7 is essentially a truth table so it is just necessary fulfill the four combinations allowed by the inequality 4.6 , besides this formula implies the disjunction of the elements $d$ is equivalent to the conjunction of the variable associated to the partitioned set $a$ and the disjunction of the conjunction of the all possible pairs of variables $d_{c}$ this is quite useful given that it simplifies the definition using a logical rule.

Example 4.3 Let $A$ be a fuzzy set, a subset of the universal set $X$ whose membership function is shown in figure 4.3 . Let $\Pi=\left\{A_{1}, A_{2}, A_{3}\right\}$ be a family of subsets of $A$ whose membership functions are shown in figure $4.3 \mathrm{~b}$ Let $a$ and $\left\{a_{1}, a_{2}, a_{3}\right\}$ be the variables obtained by applying homomorphism 4.3 on $A$ and $\Pi$ respectively. $\Pi$ fulfills the conditions to be considered $\mathcal{A}$-partition of $A$.

The total crossing set is defined as $\mathcal{K}_{T}=\mathcal{K}_{12} \cup \mathcal{K}_{23}$ since the set $\aleph_{13}=\varnothing$. With a value of $\varepsilon$ defined, the variables have the following behavior in the non-empty crossing sets: for $x \in \mathcal{K}_{12}$ take the values of $a=1, a_{1}=u, a_{2}=u$ and $a_{3}=0$, hence

$$
\begin{aligned}
& d=a_{1} \vee a_{2} \vee a_{3}=u \vee u \vee 0=u \quad \text { and } \\
& d_{c}=\left(a_{1} \wedge a_{2}\right) \vee\left(a_{1} \wedge a_{3}\right) \vee\left(a_{2} \wedge a_{3}\right)=u .
\end{aligned}
$$


International Journal on Soft Computing (IJSC) Vol.12, No.2/3/4, November 2021

For $x \in \mathcal{K}_{23}$ the variables take the value from $a=u, a_{1}=0, a_{2}=u$ and $a_{3}=u$, then

$$
\begin{gathered}
d=a_{1} \vee a_{2} \vee a_{3}=0 \vee u \vee u=u \\
d_{c}=\left(a_{1} \wedge a_{2}\right) \vee\left(a_{1} \wedge a_{3}\right) \vee\left(a_{2} \wedge a_{3}\right)=u .
\end{gathered}
$$

For elements $x$ not belonging to $\aleph_{T}$ the expressions $d=a$ and $d_{c}=0$ are fulfilled.

Table 4.3 shows the truth values of the variables $a$ and $\left\{a_{1}, a_{2}, a_{3}\right\}$, as well as the $d_{c}$ and $d$ values. It can be seen that this table satisfies proposition 2 , since $\mathrm{d}$ is reproduced by the formula 4.7 .

Table 4.3. Truth table for the example 4.3

\begin{tabular}{ccc|ccc}
$a_{1}$ & $a_{2}$ & $a_{3}$ & $a$ & $d_{c}$ & $d$ \\
\hline 0 & 0 & 0 & 0 & 0 & 0 \\
$u$ & 0 & 0 & $u$ & 0 & $u$ \\
1 & 0 & 0 & 1 & 0 & 1 \\
$u$ & $u$ & 0 & 1 & $u$ & $u$ \\
0 & 1 & 0 & 1 & 0 & 1 \\
0 & $u$ & 0 & $u$ & 0 & $u$ \\
0 & $u$ & $u$ & $u$ & $u$ & $u$ \\
0 & 0 & $u$ & $u$ & 0 & $u$
\end{tabular}

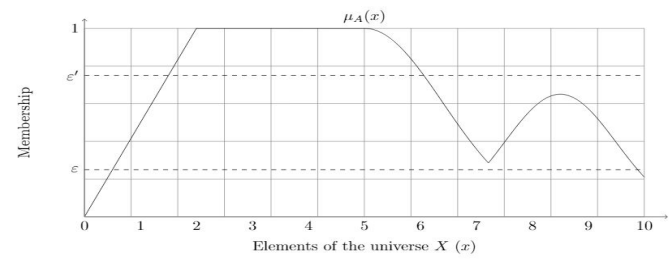

(a)

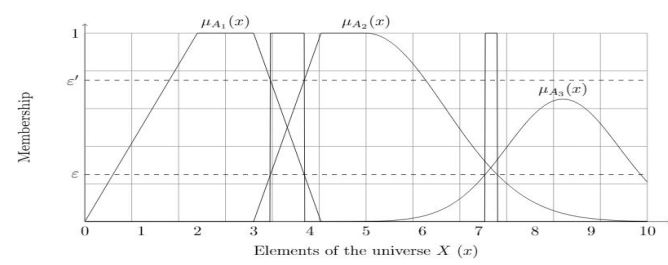

(b)

Figure 4.3. (a)Membership function $\mu_{A}(x)$ of the set to be partitioned $A$. (b)Partitioning elements $\Pi=\left\{A_{i}: i \in I\right\}$ of $A$ and their crossing sets $\mathcal{K}$.

Proposition 3 Let $\Pi=\left\{A_{i} \mid i \in I\right\}$ a finite family of $X$ fuzzy sets, then, using the standard operations.

1. Crisp partition. if $\Pi$ is a crisp partition of a subset $A \subseteq X$, then $\Pi$ is a fuzzy partition based on $\mathcal{K}_{3}$ for all $\varepsilon \in(0,0.5]$.

2. Fuzzy partitioning according to Ruspini. If $\Pi$ is a fuzzy partition according to Ruspini of a subset $A \subseteq X$, then $\Pi$ is a $\mathcal{A}$-partition for $\varepsilon \rightarrow 0^{+}$.

3. Fuzzy partitioning according to Bezdek and Harris, Dumitrescu and Ovchinikov. If $\Pi$ is a fuzzy partition according to $[6]$, one has that it is also a $\mathcal{A}$-partition for any $\varepsilon$.

4. Fuzzy partitioning according to Butnariu. If $\Pi$ is a fuzzy partition according to Butnariu of the universal set $X$, then it is also a $\mathcal{A}$-partition of the universal set $X$ for any $\varepsilon$ with $x \in \overline{K_{T}}$.

5. Fuzzy partitioning according to Markechová If $\Pi$ is a fuzzy partitioning according to Markechová then is also a $\mathcal{A}$-partition with $\varepsilon \rightarrow 0.5$.

6. Fuzzy partitioning according to Chakraborty and Das If $\Pi$ is a partition of $A$ according to Chakraborty and Das, then is also a $\mathcal{A}$-partition of $A$ for any $\varepsilon$ with $x \in \overline{K_{T}}$.

7. Fuzzy partitioning according to Bhakat and Das If $\Pi$ is a fuzzy partitioning according to Bhakat and Das, then is also a $\mathcal{A}$-partition for any $\varepsilon$. 
8. $\varepsilon$-partition If $\Pi$ is a $\varepsilon$-partition with $\alpha \in\left(\varepsilon, \varepsilon^{\prime}\right)$ of $A$, then is also a $\mathcal{A}$-partition of $A$ with $0<\varepsilon<0.5$.

\section{Proof.}

1. Since the values of the membership functions (indicator) of the crisp partitions only have the values $\{0,1\}$ it is noticeable that there are no crossing sets, so the mapping on $\mathcal{K}_{3}$ must meet the following conditions $d=a$ and $d_{c}=0$, which are equivalent to the conditions established in the crisp definition, since the algebra between crisp sets is a Boolean lattice.

2. Given the restriction on Ruspini partitions, multiple cases can be obtained;

- when $\varepsilon^{\prime}<\mu_{A_{k}} \leq 1 \Rightarrow\left\{\mu_{A_{1}}, \ldots, \mu_{A_{n}}\right\}-\left\{\mu_{A_{k}}\right\} \in[0, \varepsilon)$

- when $\varepsilon \leq \mu_{A_{K}} \leq \varepsilon^{\prime} \Rightarrow\left\{\mu_{A_{1}}, \ldots, \mu_{A_{n}}\right\}-\left\{\mu_{A_{k}}\right\} \in\left[0, \varepsilon^{\prime}\right]$

- when $0 \leq \mu_{A_{k}}<\varepsilon \Rightarrow\left\{\mu_{A_{1}}, \ldots, \mu_{A_{n}}\right\}-\left\{\mu_{A_{k}}\right\} \in(\varepsilon, 1]$

For $k=1,2, \ldots, n$.

with $\varepsilon \rightarrow 0^{+}$

- when $\mu_{A_{k}} \rightarrow 1^{-} \Rightarrow\left\{\mu_{A_{1}}, \ldots, \mu_{A_{n}}\right\}-\left\{\mu_{A_{k}}\right\} \in 0 \Rightarrow d=1$ and $d_{c}{ }^{\prime}=1$

- when $0<\mu_{A_{K}}<1 \Rightarrow\left\{\mu_{A_{1}}, \ldots, \mu_{A_{n}}\right\}-\left\{\mu_{A_{k}}\right\} \in(0,1) \Rightarrow d=u$ and $d_{c}{ }^{\prime}=u$

- when $\mu_{A_{k}} \rightarrow 0^{+} \Rightarrow\left\{\mu_{A_{1}}, \ldots, \mu_{A_{n}}\right\}-\left\{\mu_{A_{k}}\right\} \in(0,1] \Rightarrow d=0$ and $d_{c}{ }^{\prime}=0$ or $d=$ $u$ and $d_{c}{ }^{\prime}=u$

for $k=1,2, \ldots, n$. always satisfying in all cases the condition $d=d_{c}{ }^{\prime}$.

3. replacing $\triangle$ with $\wedge$ we have

$\mu_{A_{i}}(x) \wedge \mu_{A_{j}}(x)=0$

$\bigvee_{i=1}^{n} \mu_{a_{i}}(x)=1$

Then using the properties of the homomorphism $T$ we have

$a_{i} \wedge a_{j}=0$

$\bigvee a_{i}=1$

Then, given the definition 16 they are equivalent if there are no crossing sets.

4. Using the properties of homomorphisms $\bigvee_{i \in I} \mu_{A_{i}}=1 \longleftrightarrow \bigvee_{i \in I} a_{i}=1$ and $\bigvee_{j=1}^{n-1} \bigvee_{i=j+1}^{n}\left(a_{j} \wedge a_{j}\right)\left(\bigvee_{i=1}^{j} \mu_{A_{i}}\right) \wedge \mu_{A_{j+1}}=$ $0 \longleftrightarrow\left(\bigvee^{j} a_{i}\right) \wedge a_{j+1}=0$. The first expression implies $x \in \overline{K_{T}}$ according to the condition 4.4 . Using the properties of kleene algebra the second expression can be rewritten as $\left(\bigvee_{i=1}^{j} a_{i}\right) \wedge a_{j+1}=\bigvee_{i=1}^{j}\left(a_{i} \wedge a_{j+1}\right)=0$ which for each value of $j$ takes a value of 0 , that is to say that all possible disjunctions take the value of 0 fulfilling the condition 4.5 .

5. Taking into account the conditions proposed in the definition of weak partition and the standard operations, equivalent conditions can be deduced to make it a partition according to Markechová.

- $\bigvee_{i \in I} \mu_{A_{i}}(x) \geq 0.5$

- $\mu_{A_{i}} \wedge \mu_{A_{j}} \leq 0.5$ for all $i \neq j$

Given these conditions it is observable that for both to be fulfilled the intersections between fuzzy sets must become 0.5 fulfilling the definition of $\mathcal{A}$-partition for $\varepsilon \rightarrow 0.5$. 
6. The fuzzy sets with the operations supremum and infimum describe a kleene algebra, therefore, the sets can be treated as variables and the operations of infimum and supremum as the operations of conjunction and disjunction respectively by reducing the expressions to formulas 4.4 and 4.5 .

7. Given the condition $\bigcup_{i \in I} A_{i}=A$ we limit the second condition $\mu_{A_{i}}(x)+\mu_{A_{j}}(x) \leq 1$ for the case $\mu_{A_{i}} \in(0,1)$ requires that $\mu_{A_{j}}(x) \leq 1-\mu_{A_{i}}(x)$ but $\mu_{A_{j}}(x) \neq 1,0$ that is, for that point there is no cover so this definition is equivalent to the classical definition if the union is defined as the supremum.

8. By mapping the sets $A_{i}$ and $A$ we obtain the variables in $\mathcal{K}_{3} a$ and $a_{i}$ that satisfy the following implications

- $\bigcup_{i \in I} A_{i}={ }_{\varepsilon} A \Rightarrow \bigvee_{i \in I} a_{i}=a \forall a=u$

- $A_{i} \cap A_{j}={ }_{\varepsilon} \varnothing \Rightarrow a_{i} \wedge a_{j}=0$

\section{$5 \quad \mathcal{A} C$-means algorithm}

\subsubsection{Proposed solution}

The following is the $\mathcal{A}$-partition clustering method using a similar structure of the c-means algorithm.

Based on c-means, it is decided to implement a similar algorithm by changing the way the fuzzy sets are calculated, so the generated sets are not c-partitions (partitions according to Ruspini) but $\mathcal{A}$-partitions. It should be clarified that the partitions according to Ruspini are $\mathcal{A}$-partitions under the appropriate $\varepsilon$, so the algorithm proposed by Bezdek also converges to $\mathcal{A}$-partitions, but its sets are more limited in their form and conditions.

The design of the macro scale algorithm looks like this:

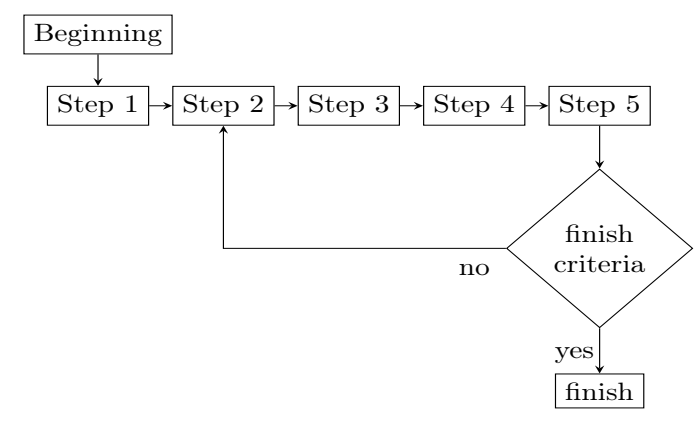

Figure 5.1. Flowchart of the c-means algorithm with $\mathcal{A}$-partitions.

\section{CLUSTERING ALGORITHM WITH $\mathcal{A}$-PARTITIONS}

The steps described below are shown as hexagons in figure 5.1

STEP 1: Pseudo-random fuzzy sets are generated within the minimum and maximum values of the input data universe.

STEP 2: The centers are calculated in exactly the same way as the conventional c-means using the equation $c_{j}=\frac{\sum_{i=1}^{D}\left(\mu_{i j}^{m} x_{i}\right)}{\sum_{i=1}^{D}\left(\mu_{i j}\right)^{m}}, \quad 1 \leq i \leq c($ taken from the c-means algorithm 28]). 
STEP 3: Kleenean values are assigned to ensure the generated sets lie in the three different possible assignments $\{0, u, 1\}$; these values are determined by taking into account the distance between the elements and the centers as follows:

1. A radius is established around the center of each of the sets, which will indicate the elements that must belong to " 1 "; due to the condition of disjoint sets, within this zone the rest of the membership values must map to " 0 ". If these n-spheres come into contact, it is established that the elements that are inside two or more belong to crossing sets and must map to " $u$ ".

2. All elements that are not inside the spheres will be determined as belonging to crossing sets and should map to " $u$ " for the two sets whose centers are closest.

For more clarity on this process see example 5.1 .

Note: This method is not unique and other $A$-partition sets can be obtained otherwise.

STEP 4: Once the kleenean mappings of the fuzzy sets are obtained, sets that fulfill these mappings are looked for, taking into account a given $\varepsilon$. It is evident that the family of sets that fulfill these conditions is infinite due to $T$ is not bijective, therefore the method chosen is one of many and tries to ensure that the generated sets are continuous. To determine the degree of membership of an element, functions that depend on the distance between the element in question and the fuzzy set to which it belongs are used. A different function is implemented for each of the possible kleenean values.

- For $a_{i}=0$

The membership function is established by taking into account the distance of the element $x$ from the radius and the largest of the distances of the centers where the kleenean assignment for $x$ is $u$.

$$
\mu_{A_{i}}(d i s)=\varepsilon\left(\frac{1}{1-A m}\left(\frac{d i s}{H_{g}}-1\right)+1\right)
$$

- For $a_{i}=u$

The membership function is established taking into account the distance of the element $x$ from the center to be evaluated and the smallest of the distances of the centers where the kleenean assignment for $x$ is $u$.

Two possible cases must be taken into consideration, first that the evaluated element is outside of an n-sphere or inside of 2 or more spheres, in the first case we use the equation

$$
\mu_{A_{i}}(d i s)=r \frac{2 \varepsilon-1}{r-H_{p}}\left(\frac{H_{p}}{d i s}-1\right)+\varepsilon
$$

in the second case:

$$
\mu_{A_{i}}(d i s)=\frac{2 \varepsilon-1}{1-\frac{H_{p}}{r}} \frac{H_{p}}{d i s}+\varepsilon-\frac{2 \varepsilon-1}{1-\frac{H_{p}}{r}}
$$

- For $a_{i}=1$

The distance dependent membership function is established $\mu_{A_{i}}($ dis $)$ In this case, the range of the function is known to be the distance dependent membership function is established $\left[\varepsilon^{\prime}, 1\right]$ and since it is known that the element in question is inside the sphere, it follows that $0<d i s<r$ then a parabolic conditioner is made between dis and $\mu_{A_{i}}$ such that $\mu_{A_{i}}(0)=1$ and $\mu_{A_{i}}(r)=\varepsilon^{\prime}$ using the equation.

$$
\mu_{A_{i}}(d i s)=\frac{-\varepsilon}{r^{2}} d i s^{2}+1
$$


STEP5 5: The objective function is calculated in exactly the same way as the conventional c-means using the equation $J_{m}(\mu, c)=\sum_{j=1}^{D} \sum_{j=1}^{N}\left(\mu_{i j}\right)^{m}\left\|x_{i}-c_{j}\right\|^{2}$ (objective function).

Example 5.1 (Assignment of kleenean variables in $\mathcal{R}^{2}$ ) Be a group of centers $\left\{C_{1}, C_{2}, C_{3}, C_{4}\right\}$ and the elements $\left\{x_{1}, x_{2}, x_{3}, x_{4}, x_{5}\right\}$ and an established radius of $r=2.5$ as shown in the figure 5.2

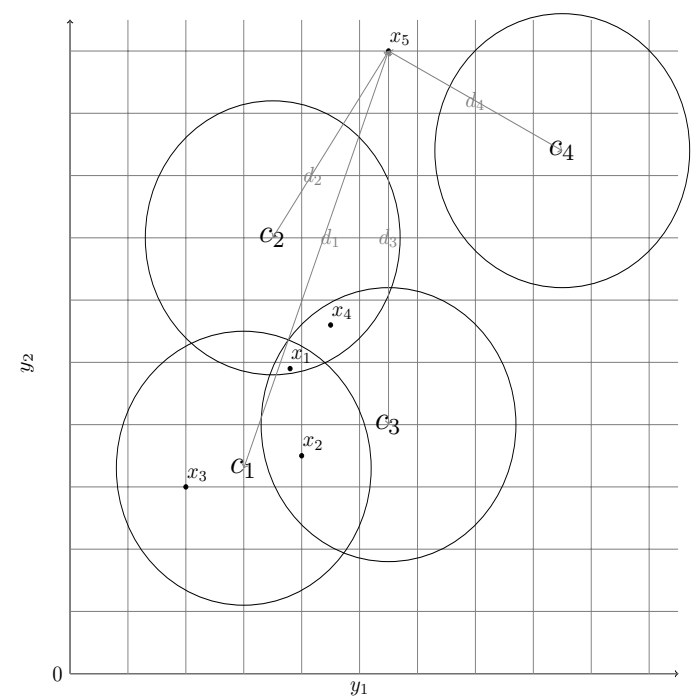

Figure 5.2. Example of assigning kleenean values to elements of an iteration

Using the criteria established in STEP 3, the kleenean assignments of the elements $x_{i}$ are determined.

Table 5.1. Kleenan assignments

\begin{tabular}{|c|c|c|c|c|c|}
\hline data & $x_{1}$ & $x_{2}$ & $x_{3}$ & $x_{4}$ & $x_{5}$ \\
\hline \multirow{4}{*}{$a_{x_{i}}$} & {$\left[\begin{array}{l}u \\
\end{array}\right.$} & {$\left[\begin{array}{l}u \\
u \\
0\end{array}\right]$} & {$\left[\begin{array}{l}u \\
0 \\
u \\
0\end{array}\right]$} & {$\left[\begin{array}{l}1 \\
0 \\
0 \\
0\end{array}\right]$} & {$\left[\begin{array}{l}0 \\
u \\
u \\
0\end{array}\right]$} \\
{$\left[\begin{array}{l}0 \\
u \\
0 \\
u\end{array}\right]$} \\
\hline
\end{tabular}

Where: $a_{x_{i}}=\left[\begin{array}{c}a_{C_{1}}\left(x_{i}\right) \\ a_{C_{2}}\left(x_{i}\right) \\ a_{C_{3}}\left(x_{i}\right) \\ a_{C_{4}}\left(x_{i}\right)\end{array}\right]$ indicating what is the kleenean value after being operated by the homomorphism of the fuzzy set whose center is $C_{n}$.

For the particular case of $x_{5}$ it is remarkable that this element is outside all the n-spheres, which indicates that the distance to the centers must be taken into account to determine the kleenean values to be taken. In figure 5.2 it is evident that the two shortest distances are $d_{2}$ and $d_{4}$, hence for the values of $a_{C 2}\left(x_{5}\right)$ and $a_{C 4}\left(x_{5}\right)$ a value of $u$ is assigned and in the rest 0 as is visible in Table 5.1

Note : The ways of determining the sets of $\mathcal{A}$-partitions are diverse and this one is chosen because of its simplicity in determining their kleenean variables centered on $c_{j}$

Example 5.2 (Determination of continuous fuzzy sets) Given three fuzzy sets with their respective centers $c_{1}=(0.3,0.75), c_{2}=(0.5,0.25), c_{3}=(0.8,0.7)$, these sets are assigned a fuzzy value taking into account a radius $r=0.2$ and a kleenean value assignment as shown in step 3 . 


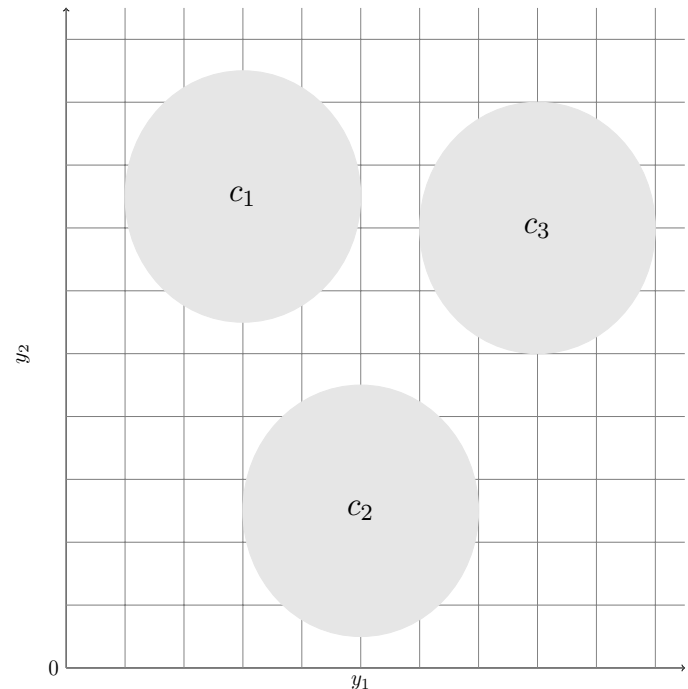

(a)

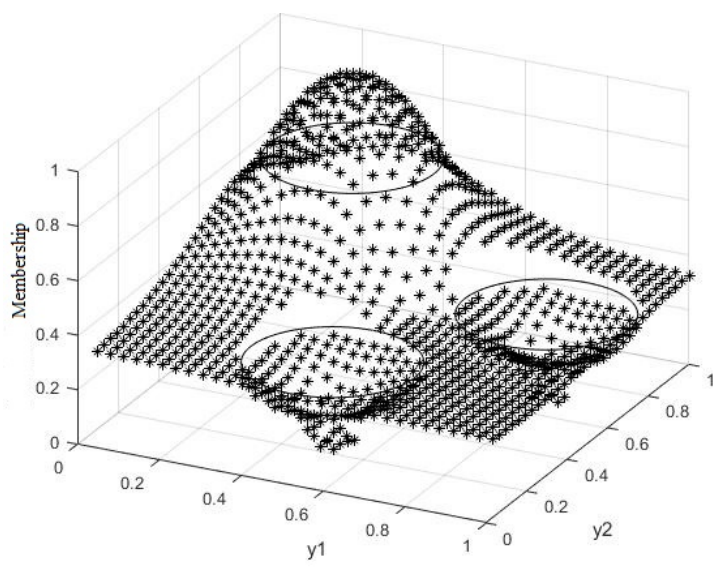

(b)

Figure 5.3. (a)Centers and radii described in this example. (b)Membership function $\mu_{c_{1}}\left(y_{1}, y_{2}\right)$ with $\varepsilon=0.25$ considering as input elements a matrix of values uniformly covering the universe $X$.

\section{Results}

Given the cluster design, it is required to vary two parameters, the $\varepsilon$ and the radius, which determine the shape of the arrays used. For this purpose, a matrix of experiments varying both parameters will be made in order to observe their optimization landscape in this particular problem.

The experiment matrix used was $21 \times 21$ in which $\varepsilon$ and $r$ were varied in the intervals [0.01, 0.49] and $[0,20]$ respectively. For each of the combinations, one hundred repetitions of the experiment were made with the purpose of finding the best test subject taking into account the objective function and the corresponding validations. In order to validate the performance of the cluster, different criteria will be used, including some internal validation metrics. The internal validation metrics are those validations that do not require data other than the data provided to the cluster and seek to validate the cohesion and separation between the data; the cohesion seeks to measure the distance between members of the same class, since a member of a cluster should be as close as possible to the other members of the same cluster and the separation measures the distance between the different clusters, since the clusters should be widely separated between them. There are several approaches to measure this distance between clusters, either distance between the closest member, distance between the most distant members or the distance between the centroids. Criteria taken from 29 .

- $F S S W=\sum_{i=1}^{k} \sum_{x \in C_{i}} \mu_{i x}\left\|m_{i}-x_{i}\right\|^{2}$ (Optimization criterion: maximum)

- $F S S B=\sum_{j=1}^{k} n_{j} \mu_{j} *\left\|c_{j}-\bar{x}\right\|^{2}$ (Optimization criterion: maximum)

- Hartigan: $H=\log \left(\frac{S S B}{S S W}\right)($ Optimization criterion: minimum) 


\section{International Journal on Soft Computing (IJSC) Vol.12, No.2/3/4, November 2021}

Table 6.1. Comparison of the internal validation results obtained with the traditional c-means method and $\mathcal{A c}$ means; in gray those criteria that were higher compared to the other algorithm.

\begin{tabular}{|l|l|l|l|l|}
\hline & $\begin{array}{c}\text { Used } \\
\text { cluster }\end{array}$ & FSSW & FSSB & Hartigan \\
\hline Cluster_n=2 & c-means & 21889.49 & 2492.323 & -1.715 \\
\cline { 2 - 5 } & Ac-means & 1362.7 & 2550.9 & -17.394 \\
\hline \multirow{2}{*}{ Cluster_n=3 } & c-means & 6050.571 & 2220.719 & -1.099 \\
\cline { 2 - 5 } & Ac-means & 2044.1 & 2651.9 & -11.7076 \\
\hline \multirow{2}{*}{ Cluster_n=4 } & c-means & 4161.423 & 2047.096 & -0.741 \\
\cline { 2 - 5 } & Ac-means & 2725.5 & 2661 & -0.7331 \\
\hline
\end{tabular}

Table 6.2. The parameters with which the best results were obtained in each of the criteria established with the different cluster_n tested

\begin{tabular}{|l|l|l|l|}
\hline Cluster_n & FSSW & FSSB & Hartigan \\
\hline \multirow{2}{*}{2} & $r=0.1$ & $r=15$ & $r=9.04$ \\
\cline { 2 - 4 } & $\varepsilon=0.01$ & $\varepsilon=0.01$ & $\varepsilon=0.01$ \\
\hline \multirow{2}{*}{3} & $r=0.1$ & $r=10.53$ & $r=12.765$ \\
\cline { 2 - 4 } & $\varepsilon=0.01$ & $\varepsilon=0.01$ & $\varepsilon=0.01$ \\
\hline \multirow{2}{*}{4} & $r=0.1$ & $r=9.04$ & $r=10.53$ \\
\cline { 2 - 4 } & $\varepsilon=0.01$ & $\varepsilon=0.01$ & $\varepsilon=0.01$ \\
\hline
\end{tabular}
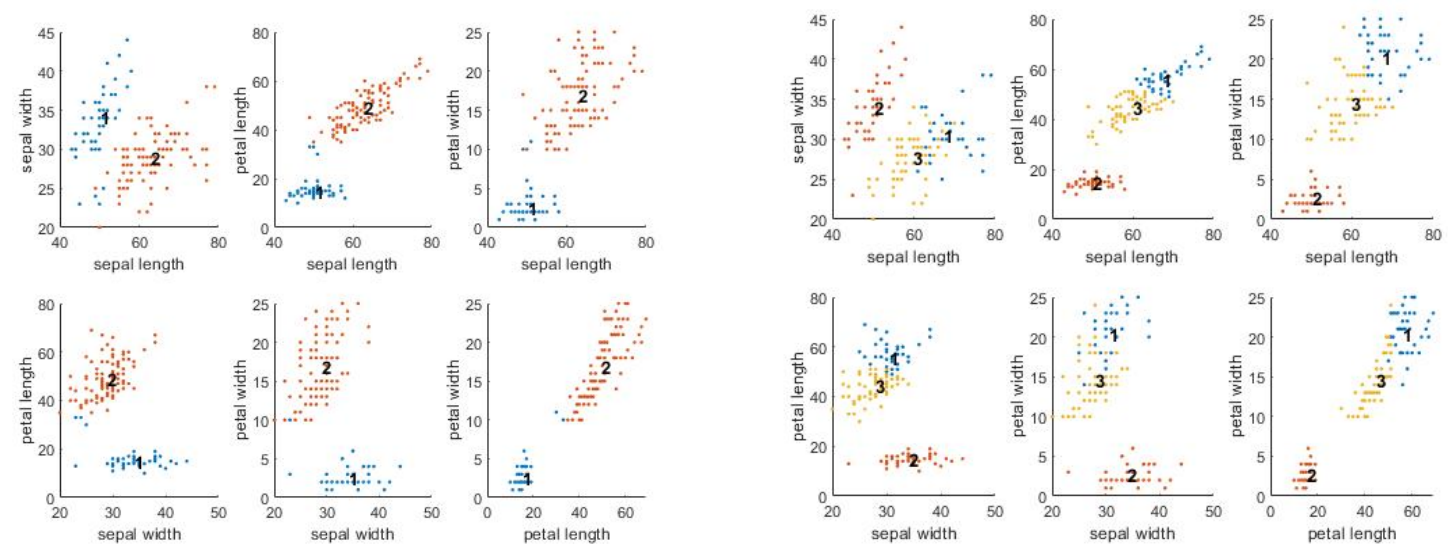

(a)

(b)
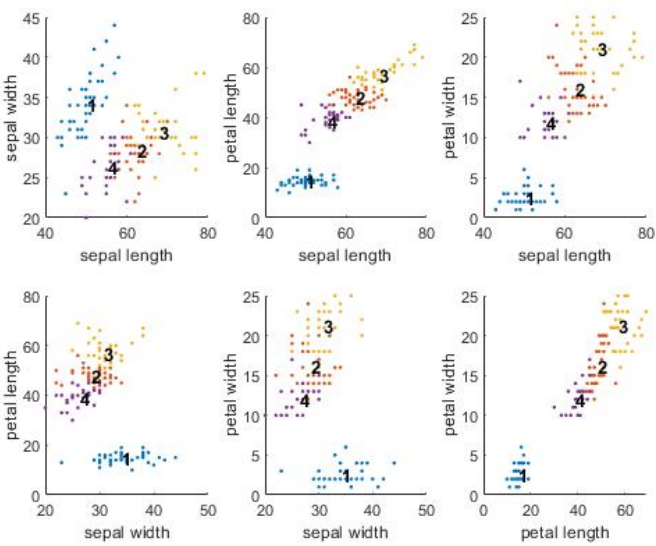

(c)

Figure 6.1. (a)Centers located by the algorithm with cluster $n=2$. (b)Centers located by the algorithm with cluster_ $n=3$. (c)Centers located by the algorithm with cluster_ $n=4$. 


\section{Discussion}

In general $\mathcal{A}$ c-means results stand out since there are a greater number of criteria in which it is better than the traditional c-means method. But it should be noted that the $\mathcal{A c}$-means has more input parameters that allow it to fit better the problem. This is not entirely convenient since having an additional parameter implies a difficulty in the search.

By observing the result of the experiments it is clear that in order to choose the input radius of the c-means with $\mathcal{A}$-partitions similar dimensions to existing clusters should be chosen for best results.

It is possible to use this proposal in some application, with the objective of comparing it with other methodologies present in the literature by making use of some previously established metrics.

Having an extra parameter allows the algorithm to be more flexible for the problem, however it increases the difficulty in finding the optimal parameters. Since it was determined that the best $\varepsilon$ are those close to 0 is not necessary that this is defined by the user, so finally the only variable input parameter of the algorithm is the value of the radius with which you want to make the clusters.

The FSSW is considered a separation metric while the FSSB is considered a cohesion metric, as it is clear in table 6.1 in both results the $\mathcal{A c}$-means is better but the last criterion, "Hartigan", is a mixed criterion that uses a cohesion measure and a separation measure, in which it is evident that the traditional method stands out.

\section{Conclusions}

A definition of fuzzy partition element was proposed which, being based on $\mathcal{K}_{3}$, allows a wide flexibility since it contains other definitions given particular conditions.

Thanks to the definition proposed in terms of $\mathcal{K}_{3}$ it was possible to use the disjunctive normal form, reducing this definition to a formula in terms of $a, d$ and $d_{c}$, using simplification methods under the distribution and absorption properties of the kleenean algebra.

The use of $\mathcal{A}$-partitions in the implementation performed, in general terms, allowed obtaining better results compared to traditional methods, in most of the performance metrics evaluated, which can be seen in table 6.1 .

This method allows a categorization of the classes using the set of elements $\{0, u, 1\}$, which makes it possible to obtain a clear and simple interpretation of the classification obtained, since instead of evaluating a degree of membership in an interval of infinite values, it can be covered in three possible algebraic values, facilitating the determination of linguistic labels.

\section{REFERENCES}

[1] E. H. Ruspini, "A new approach to clustering," Information and Control, vol. 15, pp. 22-32, 1969.

[2] H. T. Nguyen and E. A. Walker, A First Course in Fuzzy Logic, C. bibinitperiod H. /CRC, Ed. Chapman \& Hall /CRC, 2006.

[3] G. J. Klir and B. Yuan, Fuzzy Sets and Fuzzy Logic Theory and Applications, P. Hall/PTR, Ed. Prentice Hall/PTR, 1995.

[4] O. Salazar and J. Soriano, Método de simplificación de fórmulas por medio de álgebras finitas. UD editorial, 2018.

[5] L. Zadeh, "Fuzzy sets," Information and Control, vol. 8, pp. 338-353, 1965.

[6] J. C. Bezdek and J. D. Harris, "Fuzzy partitions and relations; an axiomatic basis for clustering," Fuzzy Sets and Systems, vol. 1, pp. 111-127, 1978.

[7] D. Dumitrescu, "Fuzzy partitions with the connectives $\mathrm{t} \infty$, s $\infty$," Fuzzy Sets and Systems, vol. 47, pp. 193-195, 1992. 
[8] S. Ovchinnikov, "Similarity relations, fuzzy partitions, and fuzzy orderings," Fuzzy Sets and Systems, vol. 40, pp. 107-126, 1991.

[9] D. Butnariu, "Additive fuzzy measures and integrals i," Journal of Mathematical Analysis and Applications, vol. 93, pp. 436-452, 1983.

[10] D. Markechová, "The entropy of fuzzy dynamical systems and generators," Fuzzy Sets and Systems, vol. 48, pp. 351-363, 1992.

[11] M. Chakraborty and M. Das, "On fuzzy equivalence i," Fuzzy Sets and Systems, vol. 11, pp. 185-193, 1983.

[12] S. Bhakat and P. Das, "Q-similitudes and q-fuzzy partitions," Fuzzy Sets and Systems, vol. 51, pp. 195-202, 1992.

[13] P. Quirós, P. Alonso, I. Díaz, and S. Montes, "On $\delta$ - $\varepsilon$-partitions for finite interval-valued hesitant fuzzy sets," International Journal of Uncertainty, Fuzziness and Knowledge-Based Systems, vol. 24, pp. 145-163, 2016.

[14] S. Burris and H. P. Sankappanavar, A Course in Universal Algebra. S. Burris and H.P. Sankappanavar, 2012.

[15] J. Ignjatović, M. Cirić, and S. Bogdanović, "Fuzzy homomorphisms of algebras," Fuzzy Sets and Systems, vol. 160, pp. 2345-2365, 2009.

[16] G. Grätzer, Lattice Theory: Foundation. Birkhäuser, 2011.

[17] G. Birkhoff, Lattice Theory (Colloquium Publications (Amer Mathematical Soc)). American Mathematical Society, 1940.

[18] L. H. Kauffman, "De morgan algebras - completeness and recursion," Proceedings of the Eighth International Symposium on Multiple-Valued Logic. 82-86, 1978.

[19] D. E. Muller, "Treatment of transition signals in electronic switching circuits by algebraic methods," IRE Transactions on Electronic Computers, vol. EC-8, pp. 401-401, 1959.

[20] M. Mukaidono, "Algebraic structures of interval truth values in fuzzy logic," in Proceedings of 6th International Fuzzy Systems Conference. pp. 699-705., IEEE, 1997.

[21] M. Gehrke, C. Walker, and E. Walker, "A mathematical setting for fuzzy logics," International Journal of Uncertainty, Fuzziness and Knowledge-Based Systems., vol. 05, pp. 223-238, 1997.

[22] M. Gehrke, C. L. Walker, and E. A. Walker, "Normal forms and truth tables for fuzzy logics," Fuzzy Sets and Systems, vol. 138, no. 1, pp. 25-51, 2003.

[23] J. Font and V. Verdu, "Abstract characterization of a four-valued logic," in Proceedings. The Eighteenth International Symposium on Multiple-Valued Logic., IEEE Comput. Soc. Press, 1988, pp. 389-396.

[24] K. Maes and B. D. Baets, "Facts and figures on fuzzified normal forms," IEEE Transactions on Fuzzy Systems, vol. 13, pp. 394-404, 2005.

[25] Mukaidono, "Regular ternary logic functions - ternary logic functions suitable for treating ambiguity," IEEE Transactions on Computers, vol. C-35, no. 2, pp. 179-183, Feb. 1986. DOI: $10.1109 /$ tc.1986.1676738

[26] S. C. Kleene, Introduction to Metamathematics. North Holland Publishing Co., Amsterdam, 1952.

[27] J. D. Chacón, J. Soriano, and O. Salazar, "Sistema de inferencia difusa basado en relaciones booleanas y kleeneanas con combinador convexo," Revista Ingenieria, 2017.

[28] J. C. Bezdek, R. Ehrlich, and W. Full, "FCM: The fuzzy c-means clustering algorithm," Computers \& Geosciences, vol. 10, no. 2-3, pp. 191-203, Jan. 1984. DOI: 10.1016/00983004(84) 90020-7.

[29] Métricas para la validación de clustering, https://docplayer.es/71694389-Metricas-para-lavalidacion-de-clustering.html, consulted at 22/03/20. 